\title{
Diskurs-Latenz: Re-Aktivierungen von Stereotypen rund um HIV/AIDS in Onlineforen
}

\begin{abstract}
Nicht nur die Konsequenzen einer Infektion mit dem HI-Virus - von einer tödlichen zu einer chronischen Erkrankung - haben sich seit dessen Entdeckung massiv gewandelt, sondern auch die öffentliche Auseinandersetzung damit. Nach einer kontroversen Debatte in den 1980er und frühen 1990er Jahren ist HIV/AIDS inzwischen aus der öffentlichen Wahrnehmung verschwunden. In der virulenten Diskursphase war insbesondere die Verknüpfung der Infektion mit Tod, Sexualität und Moral zentral. Im Beitrag wird am Beispiel von Postings aus dem Onlineforum med1.de argumentiert, dass Stereotype und Muster aus der frühen Phase als Diskurs-Latenzen aktuelle Konzeptualisierungen von HIV/AIDS prägen, indem die genannten Stereotype und Muster re-aktiviert werden.
\end{abstract}

Keywords: Diskurs-Latenz, HIV/AIDS, Subjektive Krankheitstheorie, Korpuspragmatik, Onlineforen

\section{$1 \mathrm{HIV/AIDS}$ damals und heute - eine Einführung}

\subsection{Der Fall Charlie Sheen - Beobachtungen zu aktuellen Konzeptualisierungen von HIV/AIDS}

His father [= Martin Sheen, DK] is a very, very decent man and a dear friend of mine. I feel bad for him. But Charlie, I don't feel bad for him. He's getting what he deserves. If you're going to misbehave like that, then they're going to get you. $\quad$ (Loose Women, 08.12.2015)

\footnotetext{
Anmerkung: Dieser Text ist im Rahmen des Forschungsprojektes Kommunikation und (Nicht-) Wissen über HIV/AIDS entstanden, das vom Schweizerischen Nationalfonds SNF mit einem Doc.CH-Stipendium (P0ZHP1_162315) und von der Universität Zürich mit einem Beitrag des Forschungskredits gefördert wurde.
}

Daniel Knuchel, Universität Zürich, Deutsches Seminar, daniel.knuchel@ds.uzh.ch 
Mit dieser Aussage reagiert Burt Reynolds in der britischen Talkshow Loose Women $^{1}$ auf die Frage, wie er das medienwirksame HIV-Outing von Charlie Sheen $^{2}$ einschätze. Insgesamt haben die Berichte über Charlie Sheen auch in Social Media große Resonanz ausgelöst, wobei viele Postings einen ähnlichen Tenor wie Reynolds Aussage aufweisen. Ein User tweetete beispielsweise: „Nonstop Hookers. Full disclosure after 4years \#HIV. \#CharlieSheen the wheels have finally come off“ (Tweet vom 17.11.2015). HIV/AIDS wird in vielen dieser Aussagen als eine Konsequenz für unangebrachtes Verhalten konzeptualisiert, also als moralisierte Krankheit, wobei eine ausgeprägte Promiskuität, Prostitution, Drogenkonsum und (potentielle) homosexuelle Handlungen zur Charakterisierung dieses „misbehaviour“ dienen. ${ }^{3}$ Als Reaktion auf solche Postings entwickelt sich aber im Mediendiskurs auch eine Gegenposition, die auf die Problematik solcher Zuschreibungen hinweist und die Diskussion um Schuld und Verantwortung als unreflektiert und vorurteilsbelastet markiert (vgl. Stenzel 2015). Die angesprochenen Vorurteile referieren auf die Verbindung von Homosexualität, Promiskuität und Drogenkonsum mit HIV/AIDS, was insbesondere den Diskurs der frühen 1980er Jahre charakterisiert (vgl. Treichler 1988).

In dieser virulenten Diskursphase war das Nachrichtenmagazin Der Spiegel für den deutschsprachigen Diskurs prägend und hat Aidserkrankungen an pauschalisierende und klischierte Vorstellungen von gelebter Sexualität gekoppelt:

Parallelen gibt es [...] zwischen den afrikanischen Phalluskultisten und den strammen schwulen Sexjägern, die [...] auf die Hatz gingen nach anonymen ,instant fucks‘ mit ständig wechselnden Partnern und in dampfenden Saunas priapische Orgien feierten. [...] Promiskuität als Motor der Seuche. Und es sieht aus, als träfe dies auch auf die Heteros von Schwarzafrika zu [...].

(Bittorf 1991: 153)

\footnotetext{
1 Burt Reynolds war am 08.12.2015 Gast in der britischen Talkshow Loose Women, um über seine Autobiographie But Enough about me zu sprechen. Er wird von den Moderatorinnen dabei auf über ihn in den 1980er Jahren zirkulierende Gerüchte angesprochen, laut denen er an AIDS erkrankt gewesen sei. Dies bildet auch den Kontext des Zitats.

2 Charlie Sheen war zu Beginn des 21. Jahrhunderts einer der bestbezahlten TV-Schauspieler in den USA und war bereits seit Ende der 1980er Jahre international berühmt und dementsprechend Thema in der (Klatsch-)Presse, wobei über seine unterschiedlichen Affären und sein Drogenkonsum berichtet wurde.

3 An dieser Stelle geht es mir nicht darum, zu negieren, dass das Risiko einer HIV-Infektion bei bestimmten Handlungen wie beispielsweise ungeschütztem Analverkehr größer ist, sondern deutlich zu machen, dass im Diskurs das HIV-Risiko aufgrund der Zugehörigkeit zu einer (devianten) Gruppe und nicht aufgrund eines Verhaltens beurteilt wird (vgl. dazu aus diskurshistorischer Perspektive Bänziger 2010).
} 
In der Gleichsetzung von schwuler und schwarzafrikanischer Sexualität werden Ausgrenzungspraktiken manifest, die typisch sind für Seuchendiskurse (vgl. Schappach 2012: 31-35). Dadurch werden (Risiko-)Gruppen konstituiert, die anders sind als die Mehrheitsgesellschaft; es entsteht ein wir gegen die anderen. ${ }^{4}$ Wie Gilman (1992: 14) herausarbeitet, werden spezifischen Menschengruppen negative Stereotype zugeschrieben, um ihnen so Verantwortung an der Entstehung und Verbreitung von (Infektions-)Krankheiten zuweisen zu können. Solche Ausgrenzungspraktiken gehen daher einher mit einer Diskussion der Schuldfrage, wie es Sontag (2003[1989]: 94) auf den Punkt bringt: „Die wenigsten fragen sich ,warum gerade ich?` Die meisten Menschen außerhalb der Subsahara-Zone wissen (oder meinen zu wissen), wie sie Aids bekommen haben.“

Weingart (2002) führt bei diesem Punkt weiter aus, dass durch die diskursive Schaffung der Kategorie der ,unschuldig' Infizierten - also zum Beispiel Personen, die sich über eine Bluttransfusion oder durch Mutter-Kind-Transmission angesteckt haben - andere HIV-Positive noch stärker als Nicht-Opfer konstruiert werden. Anders ausgedrückt: Viele HIV-Positive werden durch die Thematisierung der Infektionswege deutlicher als ,selbst schuld' markiert. Eine solche Schuldattribuierung im HIV-Diskurs wird vor allem im Topos ,HIV kriegt man nicht, HIV holt man sich“ manifest. Wenn diese Unterscheidung von ,unverschuldeten' und ,selbst verschuldeten“ Infektionen auf heute übertragen wird, zeigt sich, dass die Kategorie der ,unschuldig‘ Infizierten in der westlichen Welt höchstens noch eine marginale Rolle spielt. Dies wird beschrieben als eine Konsequenz des besseren Verständnisses der Infektionswege und daraus abgeleiteten Maßnahmen, wie zum Beispiel dem Testen von Blutspenden auf HIV oder einer intensiven Betreuung von schwangeren HIV-positiven Frauen. Auch im medial verhandelten Skandal um Charlie Sheens HIV-Infektion wird die Verfestigung der Schuldattribuierung sichtbar: Eine positive Diagnose ist selbst verschuldet, da die Übertragungswege bekannt sind und es sich daher um eine absehbare Folge eines bestimmten Verhaltens handelt. Das Beispiel illustriert zudem, dass insbesondere individuelle Vorstellungen zu HIV/AIDS - manifest in auf Social Media geteilten Reaktionen - nach wie vor mit den oben skizzierten moralischen Aspekten verbunden sind. Eine positive Diagnose ist noch immer mit einem Stigma behaftet (vgl. Stürmer \& Salewski 2009).

Aktuelle Konzeptualisierungen sind also im Fall rund um Charlie Sheen und auch ganz allgemein - von den Konzeptualisierungen der virulenten Phase des Diskurses um HIV/AIDS in den 1980er und 1990er Jahren geprägt und damit

4 Vgl. Bänziger (2014) zur Konzeptualisierung von HIV als eine Krankheit der (sexuell) Anderen. 
gleichzeitig losgelöst von aktuellen medizinischen und epidemiologischen Eigenschaften von HIV/AIDS.

\subsection{Der aktuelle Stand im Umgang mit HIV/AIDS}

In westlichen Industriestaaten ist seit 1996 eine effektive Therapie verfügbar, was die Konsequenzen einer Infektion mit dem HI-Virus grundlegend verändert hat. Mit der passenden Therapie haben HIV-positive Menschen beinahe die gleiche Lebenserwartung wie HIV-negative Menschen (vgl. von Braun et al. 2014). Es handelt sich biomedizinisch also um eine chronische Infektionskrankheit, ähnlich anderen chronischen Erkrankungen. Diese Entwicklungen markieren einen Wendepunkt, der auch diskursiv so konstruiert wird: Es gibt ein AIDS vor und eines nach der Einführung der antiretroviralen Therapie (vgl. Dannecker 2000; Knuchel 2019). In diesem Zusammenhang wird von einem Normalisierungsprozess gesprochen, in dessen Verlauf HIV/AIDS in den (klinischen) Alltag integriert worden ist und nicht mehr als außerordentlich bewertet wird. Ähnliche Bewertungen arbeitet auch Eitz (2003: 234) für den öffentlichen Diskurs Mitte der 90er Jahre heraus: HIV/AIDS habe wie jede Krankheit je eigene Spezifika, aber der Außergewöhnlichkeitsfaktor spiele kaum mehr eine Rolle. Dies lässt sich auch am verringerten Nachrichtenwert von HIV/AIDS ablesen (vgl. Lemke \& Merz 2018).

Neben der Einführung der antiretroviralen Therapie gilt die Publikation des sogenannten EKAF-Statements und die damit verbundenen Änderungen in der Präventionsarbeit als „Game-Changer“ (Patel, Curoe \& Chan 2020): Die Erfahrungen mit der effektiven antiretroviralen Behandlung von HIV-Positiven haben die Eidgenössische Kommission für Aidsfragen (= EKAF) 2008 dazu veranlasst, festzuhalten, dass „[e]ine HIV-infizierte Person ohne andere STD [= sexuell übertragbare Krankheiten, DK] unter einer antiretroviralen Therapie mit vollständig supprimierter Virämie [= Viruslast, DK] [...] sexuell nicht infektiös“ (Vernazza et al. 2008) ist. Der Botschaft des Statements - die mittlerweile durch Studien gestützt (vgl. Vernazza \& Bernard 2016) ist - wird eine stark entstigmatisierende Wirkung zugesprochen, was sich günstig auf den gesellschaftlichen Umgang mit HIV und HIV-Positiven auswirken dürfte. Wie die Reaktionen auf Charlie Sheens Outing aber vermuten lassen, hat sich der angenommene entstigmatisierende Effekt (noch) nicht entfaltet. Es bleibt daher offen, wie HIV/AIDS aktuell konzeptualisiert wird und inwiefern Fragen nach Moral, Schuld und Verantwortung gerade im alltäglichen Diskurs - damit verbunden sind.

Hier knüpft die vorliegende Untersuchung an, in der ich aktuelle Konzeptualisierungen von HIV/AIDS in einem Onlineforum in den Blick nehme. Ich arbeite 
dabei die Re-Aktivierung als konstitutives diskursives Muster einer sogenannten Diskurs-Latenz ${ }^{5}$ bei der Konzeptualisierung von HIV/AIDS heraus. Im Zentrum stehen also nicht, wie in vielen diskursanalytischen Arbeiten üblich, Diskurse zu gesellschaftlich oder politisch brisanten Themen oder zu den Diskursphasen, in denen die Brisanz bestimmter Themen eine Rolle spielt, sondern gerade die darauf folgende Zeitspanne. Es geht also um die Frage, was mit Diskursobjekten geschieht, wenn diese als nicht mehr brisant bewertet und nicht mehr in derselben Intensität öffentlich diskutiert werden.

\section{Medizinische Onlineforen und Korpuspragmatik}

\subsection{Theoretisch-methodischer Hintergrund}

Um einen Einblick in die gesellschaftlich geprägten alltäglichen Konzeptualisierungen von HIV/AIDS zu erhalten, eignen sich Diskussionen in Onlineforen als Datengrundlage, da sich im Themenbereich Gesundheit ein breitgefächertes Angebot etabliert hat, das rege genutzt wird. So gibt es einerseits Foren und Portale zu Gesundheitsthemen allgemein und andererseits spezifische Foren, die sich direkt an Betroffene einer bestimmten Krankheit richten (Rossmann \& Stehr 2019). Kleinke (2015) modelliert Diskussionen auf Onlineforen als Lai`innendiskurse, in denen insbesondere Erfahrungswissen eine zentrale Rolle spielt. Für User`innen - sowohl Schreibende als auch Lesende - hat die Kommunikation auf Gesundheitsforen verschiedene Funktionen. Sie informieren sich über Gesundheitsthemen, um dabei Wissen zu gewinnen, zu aktualisieren oder zu vertiefen. Sie interagieren, um Unsicherheiten zu klären, sich zu vergewissern und andere an ihrem Wissen teilhaben zu lassen. Zudem nutzen sie Foren für therapeutische Zwecke, um ihre Befindlichkeit zu diskutieren, Zuspruch zu erhalten und zu geben sowie die eigene Erfahrung mit anderen $\mathrm{zu}$ erörtern. In all diesen kommunikativen Handlungen manifestiert sich Wissen über Krank-/Gesundheit im Sprachgebrauch, über dessen Analyse wiederum subjektive Vorstellun-

\footnotetext{
5 In meinem Dissertationsprojekt (vgl. Knuchel i.V.) gehe ich der Frage nach, was mit einem diskursiv-kommunikativ etablierten Objekt geschieht, wenn das öffentliche Interesse daran schwindet. Auf Grundlage einer korpuspragmatischen Untersuchung zu HIV/AIDS in Zeiten der sexuellen Nicht-Infektiosität (2008-2018) konzeptualisiere ich Diskurs-Latenz dabei als Formationsprinzip, das Diskurse prägt und wiederum selbst diskursiv geprägt ist (vgl. Linke \& Schröter 2018: 454 u. 459-469).
} 
gen zu Krank-/Gesundheit ${ }^{6}$ zugänglich sind. Solche subjektiven Vorstellungen sind diskursiv geprägt und in der Musterhaftigkeit verschiedener subjektiver Vorstellungen lassen sich überindividuelle Konzeptualisierungen und letztlich auf einer abstrakteren Ebene - kollektives Wissen rekonstruieren. Bezogen auf die vorliegende Untersuchung heißt dies, dass mittels der Analyse von Postings, in denen subjektive Vorstellungen zu HIV/AIDS sprachlich manifest werden, auch Aussagen zum kulturell und diskursiv geprägten Wissen rund um HIV/ AIDS gemacht werden können.

Den theoretisch-methodischen Referenzrahmen bildet hierbei die Korpuspragmatik, wie sie für kultur- und diskursanalytische Forschung fruchtbar gemacht worden ist (vgl. Bubenhofer \& Scharloth 2015). Zentral ist die Annahme, dass Sprachgebrauchsmuster Indizien für sozial relevantes Handeln sind (vgl. Linke 2011) und mittels der Analyse solcher Sprachgebrauchsmuster auch Aussagen über gesellschaftliche Diskurse und kulturelle Praktiken sowie die sich darin manifestierenden Konzeptualisierungen von z. B. Krankheiten gemacht werden können. Mittels korpuspragmatischer Methoden lassen sich solche Sprachgebrauchsmuster berechnen und im Anschluss kulturanalytisch deuten (vgl. Bubenhofer 2009). Dabei werden datengeleitete Verfahren (wie z. B. Keywords oder Kollokationen) mit hermeneutischen Lektüren von Konkordanzen (Textausschnitte) kombiniert, um so der Komplexität eines sprachlich verhandelten Phänomens gerecht zu werden. Ein korpuspragmatisches Vorgehen erlaubt also ein neues digital-kulturphilologisches Lesen, ${ }^{7}$ da die klassischen Ausgangstexte - in der vorliegenden Untersuchung Diskussionen in Webforen - in neue Texte wie etwa Wort- und Textausschnittlisten sowie Visualisierungen transformiert ${ }^{8}$ werden.

\footnotetext{
6 Subjektive Vorstellungen zu Krank-/Gesundheit sind Gegenstand unterschiedlicher Disziplinen, die sich am Konzept der Subjektiven Theorien orientieren. Subjektive Krankheitstheorie (= SKT) meint hierbei nach Faller (1990: 53) ,die gedankliche Konstruktionen Kranker über das Wesen, die Verursachung und die Behandlung ihrer Erkrankung“. Wie Birkner \& Vlassenko (2015: 140) im Anschluss daran betonen, haben auch Gesunde mentale Repräsentationen von Krankheiten, diese sind bisher aber nicht im Fokus der Erforschung von SKT. Zu Subjektiven Krankheitstheorien von HIV-positiven Menschen vgl. Vlassenko (2015).

7 Unter dem Label Kulturphilologie ist eine Arbeitsgruppe von Nachwuchswissenschaftler^innen an der Universität Zürich tätig - mit dem Ziel, kulturwissenschaftlich grundierte Literaturund Sprachwissenschaft produktiv aufeinander zu beziehen.

8 Bubenhofer (2018: 25-26) argumentiert ähnlich, wenn er erstens davon spricht, dass Ergebnisse quantitativer Analysen neue Daten sind, die hermeneutisch gedeutet werden müssen, und er zweitens korpuslinguistisches Arbeiten als „diagrammatisches Operieren“ versteht.
} 


\subsection{Med1.de - Datengrundlage und Analysevorgehen}

Der vorliegenden Untersuchung liegen Diskussionsbeiträge des Onlineforums med1.de zugrunde. med1.de ist ein Produkt von Netdoktor und funktioniert als Online-Community zu medizinischen Themen. Es handelt sich um ein öffentliches medizinisches Online-Gesprächsforum (vgl. Kleinke 2015: 417), bei dem User`innen die Möglichkeit haben, in verschiedenen thematischen Unterforen Fragen $\mathrm{zu}$ stellen, Beiträge $\mathrm{zu}$ kommentieren und vorhandene Diskussionen (auch ohne Anmeldung) zu lesen. Es werden Informationen und Erfahrungen unter medizinischen Lai`innen ausgetauscht, so dass von typischer Peer-Kommunikation gesprochen werden kann. Diskussionseröffnende Beiträge richten sich in der Regel an eine große Bandbreite von User*innen, so dass insbesondere Erfahrungswissen im Zentrum des Austausches steht. Auch wenn Userinnen häufig einen Bezug zu den diskutierten Gesundheitsthemen haben, handelt es sich nicht per se um ein klassisches Betroffenenforum. Die User`innen, die sich im Forum äußern, können also - müssen aber nicht - einen besonderen Bezug zu den diskutierten Krankheiten haben. In den Diskussionen manifestiert sich so eine große Varianz an diskursiv geprägten Vorstellungen zu den Krankheiten im vorliegenden Beispiel zu HIV/AIDS.

Im Rahmen meines Dissertationsprojektes habe ich das Teilkorpus Persönliche Diskursarena aufgebaut, das in der hier verwendeten Version (V1.0) alle öffentlich zugänglichen Diskussionen auf med1.de beinhaltet, die bis ins Jahr 2015 geführt wurden. Das gesamte Forum wurde mithilfe eines Python-Scripts gecrawlt und anschließend korpuslinguistisch ${ }^{9}$ aufbereitet, so dass mit dem Interface CQPWeb (vgl. Hardie 2012) verschiedene Abfragen und Analysen durchgeführt werden können. Insgesamt weist das Korpus 71.969 Texte (= Diskussionsstränge) mit rund 120 Millionen Token (= laufende Wortformen) auf und dient bei verschiedenen Analysen zugleich als Referenzkorpus. Für die vorliegende Untersuchung wurde ein Subkorpus aus allen Diskussionen gebildet, die erstens zum Subforum HIV gehören und zweitens einen der beiden Suchausdrücke Verantwortung resp. Schuld oder Komposita daraus beinhalten. ${ }^{10}$ Insgesamt wurden 325 Diskussionen eingeschlossen, so dass das Untersuchungskorpus aus 8.696 Postings mit insgesamt

9 Die Texte wurden in eine XML-Struktur überführt und mit dem TreeTagger lemmatisiert und POS-getaggt.

10 Das Subforum HIV beinhaltet insgesamt 1.248 Diskussionen, die zwischen 2003 und 2015 eröffnet wurden. Die Diskussionen bestehen aus 7.173 Postings. 
759.043 Token besteht. ${ }^{11}$ Die Diskussionen bestehen im Durchschnitt aus 26,7 Postings (Median = 20; $\operatorname{Min}=1$; $\operatorname{Max}=222$ ).

Um die spezifischen Konzeptualisierungen von HIV/AIDS herauszuarbeiten, kombiniere ich quantitative und qualitative Verfahren. In einem ersten Schritt werte ich Keyword-Listen ${ }^{12}$ aus, um so wortschatzbasiert die Kontexte der Diskussionen $\mathrm{zu}$ rekonstruieren. Dabei werden die Kollokationen ${ }^{13}$ und Konkordanzen (= Textausschnitte geordnet nach Suchwort) der Keywords qualitativ ausgewertet, um die inhaltliche Relevanz der Ausdrücke zu prüfen. In einem nächsten Schritt werden Beobachtungen aus der Konkordanzanalyse als Ausgangspunkt genommen, um nach unterschiedlichen Ausprägungen bestimmter diskursiver Muster zu suchen und so die Charakteristika der Konzeptualisierungen von HIV/ AIDS mit Bezug auf Stereotypisierung und Moralisierung herauszuarbeiten. Die Analyse bewegt sich also je nach Analyseschritt zwischen quantitativen Zugängen und qualitativer Tiefenanalyse.

\section{Vermeintliche Risikosituationen, Symptome und deren Bewertung - die thematische Einbettung von HIV/AIDS}

Im Subforum HIV werden unterschiedliche Diskussionen rund um das Thema HIV geführt. Im Initialposting wird in der Regel eine Frage gestellt, wobei der Detaillierungsgrad der Schilderung und damit auch die Fülle an Kontextinfor-

11 Der Diskussionsstrang HIV-Plauderfaden ist ausgeschlossen worden, da er mit der Idee eröffnet worden ist, einen Raum anzubieten, in dem HIV/AIDS nicht das Thema sein soll und er zudem aufgrund seiner Größe (= rund ein Drittel des Subkorpus) zu dominant wäre für die statistischen Analysen.

12 Mit einer Keywordanalyse wird das typische Vokabular des Untersuchungskorpus im Vergleich zu einem Referenzkorpus berechnet. Dazu werden die Frequenzen aller Wörter beider Korpora ausgezählt und mittels eines Signifikanztests bestimmt, welche Wörter überzufällig häufig vorkommen (vgl. McEnery 2016; zu statistischen Massen: Gabrielatos 2018). In der vorliegenden Untersuchung wurde mit dem Loglikelihood-Wert $(\mathrm{p}<0,000001)$ gearbeitet. Wichtig: Die so gefundenen Keywords sind statistische Keywords, die erst in einer qualitativen Analyse auch auf ihre semantische Salienz hin geprüft werden.

13 Kollokationen sind „Paare von Worteinheiten [...], die innerhalb einer bestimmten Distanz zueinander kookkurieren und eine statistisch feststellbare Bindung zueinander aufweisen“ (Bubenhofer 2017: 69). In CQPWeb ist es möglich, eine Kollokationsanalyse durchzuführen, wobei diese in der vorliegenden Untersuchung auf Lemmabasis und mit Loglikelihood-Statistik durchgeführt wurde. 
mationen variieren kann. In den Diskussionen werden verschiedene Themen aufgebracht, wobei die Keywordanalyse für alle 8.696 Postings unterschiedliche Schwerpunkte sichtbar macht. Mithilfe von CQPWeb (Lemma basiert; Referenzkorpus $=$ med1.de komplett; Loglikelihood-Statistik mit Cut Off 0,001 \%) sind 606 Keywords berechnet worden. 39 der Keywords stellen die Pseudonyme einzelner Schreiber*innen dar: Es handelt sich also um Schreiber`innen, die sich häufig in den untersuchten Diskussionen äußern und von anderen Schreiber`innen direkt angesprochen oder als Urheberinnen einer Aussage referenziert werden.

Sehr präsent wird über die Möglichkeiten diskutiert, sich auf HIV testen zu können. Es wird einerseits unspezifisch von Test, ${ }^{14}$ Hiv-test, Aidstest oder einfach testen gesprochen, andererseits aber auch sehr spezifisch über Testverfahren wie Elisa, Western Blot, PCR, Heimtest, Antikörpertest und Schnelltest. Dabei wird detailliert erklärt, ob ak (= Antikörper) oder das p24-antigen gemessen werden, was das Ergebnis reaktiv, positiv und negativ bedeutet und welche Zeitfenster beachtet werden müssen, um diagnostische Lücken mitzudenken. Ebenfalls werden andere Infektionskrankheiten wie Tuberkulose und Malaria sowie weitere STI (= sexually transmitted infections; ein Synonym für STD) wie Chlamydien, Hepatitis, Tripper, Syphilis und Herpes genannt. Solche sexuell übertragbaren Krankheiten werden in Reaktionen auf Initialpostings thematisiert, um ganz generell auf potentielle Infektionen beim Sex hinzuweisen. Ein Großteil der Initialpostings beinhaltet die Schilderung eines Erlebnisses, um damit das potentielle Risiko einer Infektion mit HIV abzuklären. Solche vermeintlichen Risikokontakte werden detailliert besprochen, wobei der Vorfall aus unterschiedlichen Beweggründen erzählt wird. Es finden sich Postings, die direkt nach der angenommenen Risikosituation - oftmals eine Form des (Geschlechts-)Verkehrs - verfasst werden. In der Regel wird detailliert geschildert, was genau mit wem gemacht wurde: Oralverkehr, Analverkehr, Vaginalverkehr, Spermaaufnahme.

Auf lexikalischer Ebene fällt auf, dass diese Ausdrücke zum Vokabular der Sexualkunde gehören. Es wird nicht von Sex gesprochen, sondern von Verkehr, was gerade im Kontext von HIV/AIDS ein Hinweis auf eine Tabuisierung resp. Scham beim Schildern sexueller Vorlieben und Praktiken darstellen könnte. Neben Verkehr finden sich auch Ausdrücke aus dem medizinischen Fachwortschatz wie Vaginalsekret, Scheidensekret, Scheidenflüssigkeit oder Körperflüssigkeit. Einerseits gewinnen Schilderungen durch die Verwendung von Fachausdrücken an Präzision; so wird bspw. mit Verkehr stärker auch die mechanische Reibung respektive der Austausch von Körperflüssigkeiten betont. Andererseits bietet der Fachwort-

14 Kursiv gesetzte Ausdrücke sind zitierte Keywords aus dem Korpus. 
schatzgebrauch aber auch die Möglichkeit, sich sprachlich zu distanzieren. Neben der direkten Schilderung der vermeintlichen Risikosituation ist auch das Beobachten von bestimmten Symptomen am eigenen Körper Ausgangspunkt vieler Diskussionen, wie etwa im folgenden Beispiel:

Jetzt [...] geht's mir wieder richtig scheisse, ich habe Schüttelfrost, zum Abend hin immer extrem heisse Stirn/Fieber und ich bekomme andauernd hautausschlag, auf den armen und Schulter/Brust, diese verschwinden nach kurzer Zeit wieder, es handelt sich um kleine Bläschen teilweise auch längere Miteinander verbundene Pickel/Bläschen ...

Bsp. 1: Reaktionsposting | Mit HIV angesteckt?:

31_20150418_HT_702576 $6^{15}$

Symptome werden generell als grippeähnlich beschrieben oder aber detailliert aufgezählt: geschwollene Lymphknoten, näßender (Haut-)Ausschlag, Durchfall, Halsschmerz, Nachtschweiß und Mundsoor. Sie werden auch in Reaktionspostings weiter vertieft und detaillierter beschrieben, um das Risikoempfinden stärker zu legitimieren. Engagierte Schreiber`innen, die sich in vielen Diskussionen äußern und auch von anderen als Expert^innen explizit markiert werden, weisen immer wieder darauf hin, dass es sich um unspezifische Symptome handelt und sie deswegen nicht als indexikalische Zeichen für eine HIV-Infektion gelesen werden können. Die geschilderten Risikosituationen werden von den respondierenden Schreiber*innen kritisch eingeordnet:

Wie Dir (PSEUDONYM1) und (PSEUDONYM2) schon gesagt haben, hattest DU KEINERLEI Risikokontakt. OV ist safe, solange keine DEUTLICH sichtbaren Mengen von Blut im Spiel sind und solange KEIN Sperma aufgenommen wird. Also hattest Du KEINEN RK.

Bsp. 2: Reaktionsposting | Bitte um Rat / Meinung erfahrener Menschen

31_20150607_HT_705423

In vielen Diskussionen werden solche von Schreiber*innen als Risikosituation eingestufte Erlebnisse von anderen Schreiber`innen entschärft und als nicht risikoreich bewertet. Dabei wird sehr explizit und graphisch markiert (,solange KEIN Sperma aufgenommen wird“) erklärt, wieso die Einschätzung der Erlebnisse nicht stimmt, wobei kommunikativ auf Autoritäten wie als Expert^innen wahrgenommene User^innen (vgl. Bsp. 2), Institutionen wie die Aidshilfen resp. HIV-Schwerpunktpraxen oder die von Moderator`innen im Unterforum HIV und AIDS angelegten Leitfäden ${ }^{16}$ verwiesen wird. Neben Keywords, die deutlich auf

15 Die Beispiele aus dem Korpus sind alle in Originalschreibung wiedergegeben. Aus Gründen der Leserlichkeit habe ich darauf verzichtet, Fehler zu markieren. Diese Anmerkung gilt für alle Beispiele.

16 Die aktive Forumscommunity hat über Jahre Wissen zu HIV/AIDS gesammelt und für Forumsnutzer^innen zusammenfassend in Leitfäden dargestellt. Es handelt sich hierbei um ge- 
Aspekte einer HIV-Infektion im biomedizinischen Sinne verweisen, finden sich auch Keywords, die auf eine Verbindung von Moralvorstellungen und Stereotypen mit der Angst vor HIV/AIDS ${ }^{17}$ hinweisen. So wird (un-)verantwortliches Handeln thematisiert und es werden Vorurteile benannt und diskutiert, wobei Prostituierte, Homosexuelle, Drogenabhängige und Afrika eine Rolle spielen.

\section{HIV/AIDS als moralisierte Krankheit - Stereotypisierungen und Schuldzuschreibungen}

\subsection{Stereotypisierung von Risikopartner*innen}

Die Keywordanalyse zeigt, dass nebst dem Risikokontakt auch Risikogruppen eine Rolle spielen: ${ }^{18}$ Schreiber ${ }^{\star}$ innen gehen nicht nur auf sexuelle Praktiken und die Bewertung als safer sex oder ungeschützter Sex, sondern auch auf die Partner*innen in entsprechenden Situationen ein. Dabei zeigen sich verschiedene Formen der Stereotypisierung, die zum Teil auch mit expliziten und impliziten moralischen Bewertungen verbunden sind. Exemplarisch zeigt sich dies in Beispiel (3):

Nur weil das Mädel ungeschützten Verkehr hatte ist sie ja keine Virenschleuder. Außer:

1. sie arbeitet an einem Straßenstrich und hat ungesützten Verkehr mit 5-10 Männern am Tag ... das wäre was anderes.

sperrte Diskussionsfäden, die verschiedene, immer wieder auftauchende Fragen bündeln und beantworten.

17 Der Unterschied zwischen HIV und AIDS wird im Forum an verschiedenen Stellen immer wieder betont. Wie die Gebrauchsspuren (Kollokationsanalyse und Konkordanzen) von Aids und $H I V$ in den Initialpostings zeigen, setzen viele Schreibende Aids bekommen und sich mit HIV infizieren aber gleich.

18 Die diskursive Konstruktion von Risikogruppen (z. B. homosexuelle Männer) war insbesondere zu Beginn der AIDS-Pandemie zentral. So wurden im öffentlichen US-amerikanischen Diskurs, noch bevor genau bekannt war, was diese neue Krankheit auslöst und wie eine Übertragung stattfindet, vier Personengruppen als Krankheitsverbreiter konstruiert: Homosexuelle, Heroinabhängige, Haitianer und Hämophile (vgl. Tümmers 2017: 44). Der deutschsprachige Diskurs um HIV/ AIDS setzte zeitverzögert und vom US-amerikanischen Diskurs vorgeprägt ein, so dass Tümmers (2017: 55-73) von einer „importierten Angst“ spricht. Bänziger (2010, 2014, 2015) argumentiert ausgehend von dieser frühen Phase, dass der Perspektivenwechsel von Risikogruppen auf Risikopraktiken (z. B. ungeschützter Sex) ein zentraler diskursiver Wandel im HIV/AIDS-Diskurs der 1980er und 90er sei: Nicht mehr die (identifikatorische) Zugehörigkeit zu einer Gruppe ist zentral, sondern was jeweils getan oder nicht getan wird. 
2. Hatte Verkehr mit Schwule

3. Hatte Verkehr mit Junkies, die die Nadeln tauschen.

1. und 2. sind in Deutschland die größten Risikogruppen.

Bsp. 3: Reaktionsposting | Grobe Risikoeinschätzung: HIV-Übertragung bei Kondompanne 31_20130905_HT_662528

Mit dem Ausdruck Virenschleuder und durch den negativ restriktiven Konnektor außer wird stigmatisierend stereotypisiert: Nicht ungeschützter Sex an sich wird problematisiert, sondern der Kontext oder die Partnerinnen, mit denen dieser erfolgt. Im Re-Aktivieren ${ }^{19}$ der drei Kontexte Prostitution, Homosexualität und Drogenmissbrauch werden Vorstellungen zu HIV/AIDS aktualisiert, die in der frühen Phase des Diskurses geprägt wurden. Mit dem expliziten Verweis auf 5-10 Sexualpartner pro Tag als Voraussetzung einer potentiellen Infektionsgefahr wird zudem das in den 80er Jahren mit HIV/AIDS, Homosexualität und Prostitution verbundene Konzept der Promiskuität manifest. Dass dieses im obigen Beispiel nur explizit mit dem gewerblichen Sex, nicht aber mit schwulem Sex verbunden wird, ist ein Hinweis darauf, dass die diskursive Verbindung zwischen (männlicher) Homosexualität und Promiskuität nicht mehr explizit gemacht werden muss - sie wird vielmehr präsupponiert. In Beispiel 4 wird Promiskuität als typisch („die meisten schwulen“) für homosexuelle Männer markiert:

Das homosexuelle Liebesleben ist für die meisten schwulen weit fernarb jeder Monogamie und treue und moralischen vorstellungen ausgelegt...

Bsp. 4: Reaktionsposting | Abartig, krank kriminell!

31_20050214_HT_134053

Schwuler Sex als Kriterium für eine potentielle Gefahr wird an mehreren Stellen genannt; dabei gibt es direkte Thematisierungen durch eine erlebte Risikosituation (,mein RK bestand in aktivem oralsex mit einem anderen mann“ 31_20150505_HT_703534) oder indirekte Thematisierung durch das Reproduzieren von Stereotypen wie im Beispiel oben. In einem anderen Diskussionsstrang werden stereotype Vorstellungen über Homosexualität und HIV besonders deutlich: Eine Schreiberin schildert einen potentiellen Risikokontakt mit einem ihr mehr oder weniger unbekannten Mann, den sie nach einigen Treffen oral befriedigt, wobei er in ihrem Mund ejakuliert. Sie sorgt sich deswegen, was sich da-

19 Den Begriff der Re-Aktivierung (wie auch den Begriff Latenz) entlehne ich aus der medizinischen resp. mikrobiologischen (HIV-)Forschung, in der damit erklärt wird, dass reaktivierte latent infizierte Zellen (sogenannte Memory-Zellen) bei therapierten Patient^innen zu einem minimalen Anstieg der Viruslast führen können. Das Induzieren einer Re-Aktivierung solcher Memory-Zellen wird als mögliche Strategie diskutiert, um eine HIV-Infektion heilen zu können (vgl. Mann et al. 2020). 
durch verstärkt, dass er nach diesem Treffen abweisend reagiert und keinen weiteren Kontakt mehr möchte. Andere Schreiber`innen beschwichtigen die Frau in ihren Reaktionspostings und verweisen auf das äußerst geringe Risiko bei Oralsex, insbesondere wenn das Sperma nicht geschluckt werde. Ein Schreiber verweist zudem darauf, dass es weniger heterosexuelle als homosexuelle HIV-positive Männer gebe. Die Diskussionsinitiantin geht anschließend indirekt auf diese Einschränkung ein: Sie nimmt das oben geschilderte abweisende Verhalten zum Anlass, um die Ehrlichkeit des Mannes per se in Frage zu stellen und unterstellt ihm gleichgeschlechtliche sexuelle Handlungen, was sie in der Einschätzung eines erhöhten Risikos aufgrund der angenommenen Bisexualität bestärkt (31_20130119_HT_643714). Auch wenn die Schreiberin im Vergleich zu anderen Schreiber ${ }^{\star}$ innen eher eine Extremposition einnimmt, ${ }^{20}$ zeigt sich hier dennoch, wie in einer emotional belastenden Situation die stereotypisierte Verbindung zwischen HIV und Homosexualität diskurswirksam wird.

In Beispiel 3 oben werden Sexworker^innen als die zweite große Risikogruppe bezeichnet. Diese Zuschreibung wird - analog zu den homosexuellen Männern ebenfalls explizit durch geschilderte Erlebnisse wie auch implizit durch Wertungen in Nebenbemerkungen manifest. Der Kontext der Prostitution als Thema spiegelt sich auch in unterschiedlichen Keywords wider: Prostituierte (353 Treffer), Dame (289 Treffer), ${ }^{21}$ Freier (30), Bordell(besuch) (144 Treffer), Puff (44 Treffer) und Straßenstrich (15 Treffer). Wie an den absoluten Vorkommen der Ausdrücke bereits ersichtlich wird, liegt der Fokus der Diskussionen auf den Sexworker ${ }^{\star}$ innen und nicht auf deren Kund*innen. In der Mehrheit der Texte werden Erlebnisse mit Sexworker*innen auf ähnliche Weisen geschildert, so dass bestimmte Zuschreibungen musterhaften Charakter aufweisen. Grundsätzlich gehen User*innen im Forum davon aus, dass die Wahrscheinlichkeit einer HIVInfektion bei eine ${ }^{\star} m^{\star}$ r Sexworker*in sehr hoch ist, was auch metadiskursiv verhandelt wird: „Es wird ja auch grundsätzlich JEDER Prostituierten hier unterstellt HIV + zu sein“ (31_20150518_HT_704300). Dabei wird teilweise - wie auch oben in Beispiel 3 - unterschieden, in was für einem Kontext die Sexworker^innen ihre Dienste anbieten. Der Straßenstrich wird als besonders gefährlich markiert und öfters mit Drogen in Verbindung gebracht. Auch in aufklärerischen Post-

20 Im Unterforum insgesamt wird immer wieder diskutiert, inwiefern sich vor allem sogenannte HIV-Phobiker und hypochondrisch veranlagte Personen äußern, was auch in der hier zitierten Diskussion geschieht. Ob diese Zuschreibungen aber zutreffen oder nicht, ist m. E. nicht entscheidend, um die Wirkmächtigkeit der Verbindung von HIV/AIDS und Homosexualität für die Konzeptualisierung der Krankheit herauszuarbeiten.

21 Dame wird in 64 \% aller Gebrauchskontexte (191 von 289) als Bezeichnung für eine Prostituierte verwendet. 
ings, die gegen eine Markierung von Sexworker*innen als spezifische Risikogruppe anreden, wird eine innere Hierarchie sowie teilweise stigmatisierender Sprachgebrauch (Bordsteinschwalbe) deutlich:

ne, kein Risiko. Außerdem gehören Prostituierte (sofern es keine drogenabhängigen Bordsteinschwalben sind) nicht zur Risikogruppe.

Bsp. 5: Reaktionsposting | Angesteckt bei Fingerspiel durch kleine Wunde am Finger? 31_20061009_HT_268515

Neben dem Arbeitsort (Laufhaus, Bordell, Straßenstrich, Sexclub, privat via Anzeige) wird zudem die ethnische Herkunft des`der Sexworker^in relevant gesetzt:

Es war auch eine deutsche Dame gewesen die machen sich ja sicherlich mehr aus Hiv Aids wie zum Beispiel eine aus Ukraine Bulgarien ich habe halt Extremst schieß davor

Bsp. 6: Reaktionsposting | Unwissenheit und Angst

31_20150819_HT_709574

Die Herkunft von Sexualpartner^innen spielt nicht nur bei Prostituierten eine Rolle, auch wenn hier rassistische Stereotype - z. B. werden Thai-Frauen als hygienisch und deutsche Sexworker*innen als aufgeklärt und vorsichtig bewertet - besonders deutlich sprachlich manifest werden. Ganz allgemein wird insbesondere eine afrikanische oder südostasiatische Herkunft von Sexualpartner*innen zum Anlass genommen, eine HIV-Infektion zu vermuten. Gerade diese offensichtlichen Rassismen werden aber auch im Forum direkt reflektiert und kritisiert: „Als ob nur Menschen ausländischer Herkunft HIV infiziert sein können“(31_20150518_HT_704300).

\subsection{Die Zuschreibung von Schuld und Verantwortung}

Neben der Stereotypisierung von potentiellen Risikopartner*innen spielt auch die in der Einleitung skizzierte Zuschreibung von Schuld und Verantwortung eine Rolle, wodurch HIV/AIDS als Krankheit auch selbst moralisiert wird.

Es werden grundsätzlich zwei Diskussionen geführt: Auf der einen Seite wird Dritten ein eigenes Verschulden attribuiert, wenn diese erhöhte Risiken eingehen, wie z. B. mit einemr Sexworker ${ }^{\star}$ in penetrativen Sex ohne Kondom zu haben. Die fremdzugeschriebene Schuld wird auch metadiskursiv verhandelt und als dominante gesamtgesellschaftliche Interpretationsfolie herausgestellt: „[J]eder der HIV + ist, ist laut unserer verkorksten Gesellschaft selber schuld, hat er doch ohne Schutz rumgevögelt“ (31_20150518_HT_704300). In Aussagen wie diesen wird der Topos ,AIDS kriegt man nicht, AIDS holt man sich“ in sprachlich unterschiedlichen Formen realisiert. Das zeugt von einer starken Fokussierung auf die Eigen- 
verantwortung. Auf der anderen Seite wird von Schreiber`innen klar markiert, dass sie ihr eigenes ,Fehlverhalten“ einsehen:

Ich habe vor demnächst einen Test zu machen [...]. Dazu muss ich sagen bin ich selbst dran schuld und ja ich weiß, ich war einfach viel zu naiv habe mir da nie die Gedanken gemacht, weil ich die Pille genommen hatte.

Bsp. 7: Reaktionsposting | Totale Angst vor Hiv-Test, oftmals ungeschützten Sex 31_20140329_HT_677867

Dieses Posting ist exemplarisch dafür, wie der Topos ,Aids holt man sich“ sprachlich realisiert wird: Die individuelle Konzeptualisierung von HIV/AIDS ist durch den Topos beeinflusst, wird aber nicht reflektiert. Ähnliche argumentative Strukturen finden sich auch bei Konstruktionen rund um selbst verantwortlich sein.

Im Bereich der Verantwortungszuschreibung wird aber auch noch eine andere Position verhandelt:

Sie hätte mir vor unserem ungeschützten Sex eigentlich sagen sollen, dass sie früher öfters ebenfalls ungeschützten Sex hatte. Das hat sie mir aber verschwiegen und das finde ich nicht ganz ok.

Bsp. 8: Reaktionsposting | Freundin ist vielleicht HIV positiv, was soll ich tun? 31_20100101_HT_508856

Der Schreiber sieht seine Sexualpartnerin in der Pflicht, nicht nur für sich selbst, sondern auch für ihn Verantwortung zu übernehmen, da sie durch ihr Verhalten zu einer Risikopartnerin geworden sei. Dass das eigene Verhalten ebenfalls eine Rolle spielen könnte, wird an anderer Stelle vom Schreiber in der Diskussion negiert: Dies sei das erste Mal, dass er unverantwortlich gehandelt habe, sodass er den Schutz der Sexualpartnerin garantieren könne und somit seine Verantwortung ihr gegenüber wahrgenommen habe. Diese argumentative Logik des Schreibers findet sich auch sonst im Diskurs um Verantwortung bei potentiellen HIV-Übertragungen, wenn insinuiert wird, dass HIV-Positive eine größere Rolle beim Durchsetzen von Safer-Sex-Regeln spielen müssen. ${ }^{22}$ Wie auch bei den stigmatisierenden Stereotypisierungen von Risikogruppen finden sich im Forum diskursive Korrekturen zu Fragen nach der Verantwortung, wobei für eine gleichberechtigte und selbst einzulösende Verantwortung plädiert wird.

22 Bis in die 2010er Jahre war diese diskursive Position auch juristisch relevant, da potentielle HIV-Übertragungen strafrechtlich belangt wurden. Die Rechtsprechung beachtete neueres medizinisches Wissen (z. B. sowohl die Therapierbarkeit als auch die Nicht-Infektiosität bei erfolgreicher Therapie) nicht. Erst allmählich findet eine Korrektur dieser Praxis statt (vgl. zwar spezifisch für die USA, wobei einzelne Beobachtungen auch für den deutschsprachigen Raum gelten: Hoppe 2018). 


\section{Fazit - Diskurs-Latenzen und Re-Aktivierungen rund um HIV/AIDS}

In der obigen Analyse habe ich herausgearbeitet, welche Aspekte bei der Konzeptualisierung von HIV/AIDS in Postings des Subforums HIV/AIDS auf med1. de eine Rolle spielen, wenn (Selbst-)Verantwortung in irgendeiner Form thematisiert wird. Ein Großteil der Diskussionen behandelt Erlebnisse, die von den Schreiber*innen als HIV-Infektionsrisikosituationen bewertet werden, wobei in den Schilderungen sowie den Reaktionen anderer Schreiber*innen subjektive Vorstellungen zu HIV/AIDS sprachlich manifest werden. Dabei spielen Stereotypisierungen und Moralisierungen eine Rolle.

Der von Bänziger (2010) konstatierte Wandel von Risikogruppen zu Risikopraktiken wird in den Postings nur bedingt sichtbar: Unterschiedliche sexuelle Praktiken werden zwar angesprochen, aber zentrales diskursives Muster ist die Re-Aktivierung der in der frühen Phase diskursiv geprägten Risikogruppen der Homosexuellen und der Sexworker*innen. Allein die Zuordnung der Sexpartne$r^{\star}$ innen $z u$ einer dieser Gruppen reicht aus, um ein erhöhtes HIV-Infektionsrisiko anzunehmen. Zudem spielt auch die von Gilman (1992) herausgearbeitete Dichotomie zwischen wir (hier: HIV-Negative) und die anderen (hier: potentiell HIV-Positive) eine Rolle, was sich in spezifischen Schuldattribuierungen und Erwartungen an potentiell HIV-positive Personen manifestiert: Zwar wird das eigene Verhalten in vermeintlichen und potentiellen Risikosituationen kritisch reflektiert und die eigene Verantwortung und Schuld thematisiert, aber (potentiell) HIV-Positiven wird eine höhere Verantwortlichkeit für den Schutz von HIVNegativen zugeschrieben. Das eigene - vom ,korrekten' Sex abweichende Verhalten wird als Ausnahme bewertet, um das sexuelle Gegenüber als anders und damit auch als potentielle Infektionsgefahr zu konstruieren.

Es sind also Re-Aktivierungen von in der virulenten Phase geprägten Stereotypen und Mustern, die - verstanden als Diskurs-Latenzen - auch aktuelle Konzeptualisierungen von HIV/AIDS prägen.

\section{Literatur}

Bänziger, Peter-Paul (2010): Konstellationen und Koalitionen im Sprechen über Aids in den 1980er Jahren. In Achim Landwehr (Hrsg.), Diskursiver Wandel, 31-51. Wiesbaden: Springer VS.

Bänziger, Peter-Paul (2014): Vom Seuchen- zum Präventionskörper? Aids und Körperpolitik im deutschsprachigen Raum der 1980er Jahre. Body Politics 2 (3). 179-214. 
Bänziger, Peter-Paul (2015): ExpertInnen statt AktivistInnen: der Entpolitisierungsdiskurs in der Aids-Arbeit der 1980er Jahre. In Pascal Eitler \& Jens Elberfeld (Hrsg.): Zeitgeschichte des Selbst. Therapeutisierung - Politisierung - Emotionalisierung, 261-277, Bielefeld: Transcript. Birkner, Karin \& Ivan Vlassenko (2015): Subjektive Theorien zu Krankheit und Gesundheit. In Albert Busch \& Thomas Spranz-Fogasy (Hrsg.), Handbuch Sprache in der Medizin (Handbücher Sprachwissen 11), 135-153. Berlin, Boston: De Gruyter.

Bittorf, Wilhelm (1991): Mgolo ist unser Gott. Der Spiegel 25, 17. 06.1991, 146-174.

Braun, Amrei von, Hansjakob Furrer, Manuel Bettegay, Alexandra Calmy, Matthias Cavassini, Pietro Vernazza, Enos Bernarsconi, Rainer Weber \& Huldrych F. Günthard (2014): Antiretrovirale Therapie. Therapeutische Umschau 71 (8), 461-468.

Bubenhofer, Noah (2009): Sprachgebrauchsmuster. Korpuslinguistik als Methode der Diskursund Kulturanalyse (Sprache und Wissen 4). Berlin, New York: De Gruyter.

Bubenhofer, Noah (2017): Kollokationen, n-Gramme, Mehrworteinheiten. In Kersten Sven Roth, Martin Wengeler \& Alexander Ziem (Hrsg.), Handbuch Sprache in Politik und Gesellschaft (Handbücher Sprachwissen, 19), 69-93. Berlin, Boston: De Gruyter.

Bubenhofer, Noah (2018): Wenn „Linguistik“ in „Korpuslinguistik“ bedeutungslos wird. In Joachim Gessinger, Angelika Redder \& Ulrich Schmitz (Hrsg.): Korpuslinguistik (Osnabrücker Beiträger zur Sprachtheorie 92), 17-29. Duisburg: Universitätsverlag Rhein-Ruhr.

Bubenhofer, Noah \& Joachim Scharloth (2015): Maschinelle Textanalyse im Zeichen von Big Data und Data-driven Turn - Überblick und Desiderate. Zeitschrift für Germanistische Linguistik 43 (1), 1-26.

Dannecker, Martin (2000): Wider die Verleugnung sexueller Wünsche. AIDS-Infothek 1, 4-10.

Eitz, Thorsten (2003): Krankheitsgeschichte und Sprachgeschichte. Hildesheim, Zürich, New York: Olms.

Faller, Hermann (1990): Subjektive Krankheitstheorie und Krankheitsverarbeitung bei Herzinfarktrehabilitanden. Frankfurt a.M., Bern: Peter Lang.

Gabrielatos, Costas (2018) Keyness analysis: nature, metrics and techniques. In Charlotte Taylor \& Anna Marchi (Hrsg.): Corpus Approaches to Discourse. A Critical Review, 225258. London, New York: Routledge.

Gilman, Sander L. (1992): Rasse, Sexualität und Seuche. Stereotype aus der Innenwelt der westlichen Kultur. Reinbek bei Hamburg: Rowohlt.

Hardie, Andrew (2012): CQPweb - combining power, flexibility and usability in a corpus analysis tool. International Journal of Corpus Linguistics 17 (3), 380-409.

Hoppe, Trevor (2018): Punishing disease: HIV and the criminalization of sickness. Oakland: University of California Press.

Kleinke, Sonja (2015): Internetforen: Laiendiskurs Gesundheit. In Albert Busch \& Thomas Spranz-Fogasy (Hrsg.), Handbuch Sprache in der Medizin (Handbücher Sprachwissen 11), 405-422. Berlin, Boston: De Gruyter.

Knuchel, Daniel (2019): ,Old“ AIDS - ,New“ AIDS in DER SPIEGEL? A corpus linguistic approach to conceptualisations of HIV/AIDS. In Alexandra Groß, Ramona Pech \& Ivan Vlassenko (Hrsg.), HIV/AIDS: Interdisziplinäre Perspektiven (Medizin 22), 95-114. Berlin: Lit.

Knuchel, Daniel (i. V.): Diskurs-Latenzen. Korpuspragmatische Studien und kulturphilologische Diskussion zu HIV/AIDS in der Ära der Sexuellen Nicht-Infektiosität (2008-2018).

Lemke, Richard \& Simon Merz (2018): HIV/AIDS-Berichterstattung in drei Jahrzenten. Ausgewählte Ergebnisse einer Medien-Inhaltsanalyse. Mainz: Institut für Publizistik der Johannes-Gutenberg-Universität. 
Linke, Angelika (2011): Signifikante Muster: Perspektiven einer kulturanalytischen Linguistik. In Elisabeth Wåghäll Nivre, Brigitte Kaute, Bo Andersson, Barbro Landén \& Dessislava Stoeva-Holm (Hrsg.), Begegnungen. Das VIII. Nordisch-Baltische Germanistentreffen in Sigtuna vom 11. bis zum 13. 6.2009, 23-44. Stockholm: Stockholms universitet.

Linke, Angelika \& Juliane Schröter (2018): Diskurslinguistik und Transsemiotizität. In Ingo H. Warnke (Hrsg.): Handbuch Diskurs. (Handbücher Sprachwissen 6), 449-469. Berlin, Boston: De Gruyter.

Mann, Jamie F. S., Joshua Pankrac, Katja Klein, Paul F. McKay, Deborah F. L. King, Richard Gibson, Chanuka N. Wijewardhana, Rahul Pawa, Jodi Meyerowitz, Yomg Gao, David H. Canaday, Mariano Avino, Art F. Y. Poon, Caroline Foster, Sarah Fidler, Robin J. Shattock \& Eric J. Arts (2020): A targeted reactivation of latent HIV-1 using an activator vector in patient samples from acute infection. EBioMedicine 59. 102853.

McEnery, Tony (2016): Keywords. In Paul Baker \& Tony McEnery (Hrsg.): Corpora and Discourse Studies. Integrating Discourse and Corpora (Routledge Advances in Language and Linguistics), 20-32. Basingstoke: Palgrave Macmillan.

Patel, Rupa R., Katherine A. Curoe \& Philip A. Chan (2020): Undetectable Equals Untransmittable: A Game Changer for HIV Prevention. Clinical Chemistry 66 (3), 406-407.

Rossmann, Constanze \& Paula Stehr (2019): Gesundheitskommunikation im Internet. Erscheinungsformen, Potenziale, Grenzen. In Wolfgang Schweiger \& Klaus Beck (Hrsg.), Handbuch Online-Kommunikation, 393-419. Wiesbaden: Springer.

Schappach, Beate (2012): Aids in Literatur, Theater und Film. Zur kulturellen Dramaturgie eines Störfalls. Zürich: Chronos.

Sontag, Susan (2003 [1989]): Krankheit als Metapher. Aids und seine Metaphern. Frankfurt a. M.: Fischer.

Stenzel, Kendra (2015): Der Kranke als Täter. Spiegel-Online. https://www.spiegel.de/pano rama/leute/charlie-sheens-hiv-infektion-in-den-medien-a-1063190.html (letzter Zugriff: 26. 06.2020).

Stürmer, Stefan \& Christel Salewski (2009): Chronische Krankheit als Stigma: Das Beispiel HIV/AIDS. In Aandreas Beelmann \& Karl J. Jonas (Hrsg.): Diskriminierung und Toleranz, 263-281. Wiesbaden: Verlag für Sozialwissenschaften.

Treichler, Paula A. (1988): AIDS, Homophobia, and Biomedical Discourse: An Epidemic of Signification. In Douglas Crimp (Hrsg.): AIDS. Cultural Analysis. Cultural Activism, 31-70. Cambridge, London: MIT Press.

Tümmers, Henning (2017): Aids. Autopsie einer Bedrohung im geteilten Deutschland (Beiträge zur Geschichte des 20. Jahrhunderts 23). Göttingen: Wallstein.

Vernazza, Pietro \& Edwin Bernard (2016): HIV is not transmitted under fully suppressive therapy: The Swiss Statement - eight years later. Swiss Medical Weekly 146.

Vernazza, Pietro, Bernhard Hirschel, Enos Bernasconi \& Markus Flepp (2008): HIV-infizierte Menschen ohne andere STD sind unter wirksamer antiretroviraler Therapie sexuell nicht infektiös. Schweizerische Ärztezeitung 89 (5), 165-169.

Vlassenko, Ivan (2015): Sprechen über HIV/AIDS. Narrative Rekonstruktionen und multimodale Metaphern zur Darstellung von Subjektiven Krankheitstheorien (Germanistik 64). Berlin: Lit.

Weingart, Brigitte (2002): Ansteckende Wörter. Repräsentationen von AIDS. Frankfurt a.M.: Suhrkamp. 